\title{
Evolution of Cooperation through Power Law Distributed Conflicts
}

\author{
Pilwon Kim \\ Ulsan National Institute of Science and Technology (UNIST), Department of Mathematical Sciences, \\ Ulsan Metropolitan City 689-798, Republic of Korea \\ Correspondence should be addressed to Pilwon Kim; pwkim@unist.ac.kr
}

Received 24 October 2016; Accepted 20 December 2016; Published 17 January 2017

Academic Editor: Dimitri Volchenkov

Copyright (c) 2017 Pilwon Kim. This is an open access article distributed under the Creative Commons Attribution License, which permits unrestricted use, distribution, and reproduction in any medium, provided the original work is properly cited.

\begin{abstract}
At an individual level, cooperation can be seen as a behaviour that uses personal resource to support others or the groups which one belongs to. In a conflict between two individuals, a selfish person gains an advantage over a cooperative opponent, while in a group-group conflict the group with more cooperators wins. In this work, we develop a population model with continual conflicts at various scales and show cooperation can be sustained even when interpersonal conflicts dominate, as long as the conflict size follows a power law. The power law assumption has been met in several observations from real-world conflicts. Specifically if the population is structured on a scale-free network, both the power law distribution of conflicts and the survival of cooperation can be naturally induced without assuming a homogeneous population or frequent relocation of members. On the scale-free network, even when most people become selfish from continual person-person conflicts, people on the hubs tend to remain unselfish and play a role as "repositories" of cooperation.
\end{abstract}

\section{Introduction}

Understanding the survival of cooperation in the context of Darwinian evolution has been a challenge to researchers from various fields of natural and social sciences $[1,2]$. During the past decades, a variety of mechanisms have been proposed to explain how cooperation can be evolutionarily advantageous under pressure of competitive selection. Typical examples include group selection [3-5], kin selection $[6,7]$, direct/indirect reciprocity $[8,9]$, altruistic punishment $[1,10,11]$, other-regarding preferences [12-14], the social heuristic hypothesis [15], and the interdependence hypothesis [16].

At an individual level, cooperation can be seen as a behaviour that uses personal resources to benefit other individuals or a group at its own expense. When people are engaged in a conflict, the effect of cooperation may depend on the conflict scale. If a cooperator is engaged with a defector in an interpersonal dispute, then he or she is generally at disadvantage compared to the opponent as selfish opponents can take a full advantage of their resources.
In case of a large conflict between groups, on the contrary, the group with more cooperators is subject to win [17]. Thus frequent occurrence of group-level conflicts would explain the survival of cooperation. However, since such large conflicts overwhelming interpersonal disputes are relatively rare in nature, there should be more elaborate explanation on the relations between the evolution of cooperation and the scale of interactions.

In order to solve this puzzle, there have been extensive studies made in the framework of evolutionary game theory. One of the leading paradigms is the spatial prisoner's dilemma game $[18,19]$. Social diversity and stochastic variations in payoff in the spatial prisoner's dilemma game can promote cooperation greatly [20-22]. Various types of heterogeneity also haven been introduced in the population structure to affect the evolution of cooperation. In [23-25], it is shown that cooperation becomes a dominating trait among individuals if they interact following a scale-free network or other complex networks of contacts. The public goods game on the bipartite graphs has been studied as a model of collective interactions among a structured population [26, 


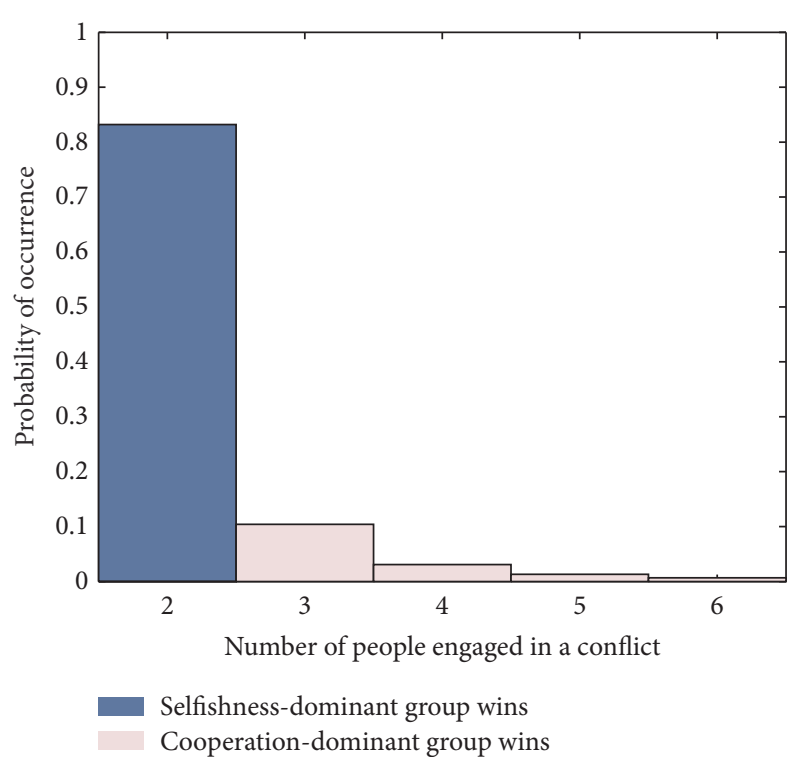

FIGURE 1: Illustration of probability of occurrence of a conflict involving $x$ persons and its consequence: conflicts are generated to be consistent with a power law, and their consequences are classified according to their size, where $k_{c}=2$.

27]. In particular, the cooperation thrives when the multilevel public goods game is played in hierarchical groups. More in-depth discussion and various examples can be found in $[28,29]$.

In this work, we develop a conflict-based population model that describes the evolution of cooperation. We assume that cooperators and defectors interact at various scales, and the size of those engagements follows a power law distribution. A claim that distribution of conflicts often resembles a power law has been raised from historical observation on violent events [30-33]. The frequency of conflict occurrence, ranging from homicides through interstate battles to international wars, is found to be inversely related to their magnitude by means of a power law, as

$$
p_{\mathrm{cft}}(x) \sim \frac{1}{x^{\alpha}}
$$

Similar patterns fitted to a power law distribution were also found in many different types of conflicts in domestic/international politics and labor strikes. In those researches on human conflict distributions, the exponent $\alpha$ estimated from the empirical data mostly ranges from 1.5 to $2.5[30,33-$ 38]. An example of power law conflict distributions is visualized in Figure 1, by which selfish people win conflicts in most cases. Suppose, for each conflict occurring according to a power law distribution (1), the people engaged in the conflict are randomly chosen from a population. Figure 2 shows a conditional probability of an individual being engaged in a large conflict when he or she happens to be chosen. As the exponent $\alpha$ increases, the tail of the distribution becomes

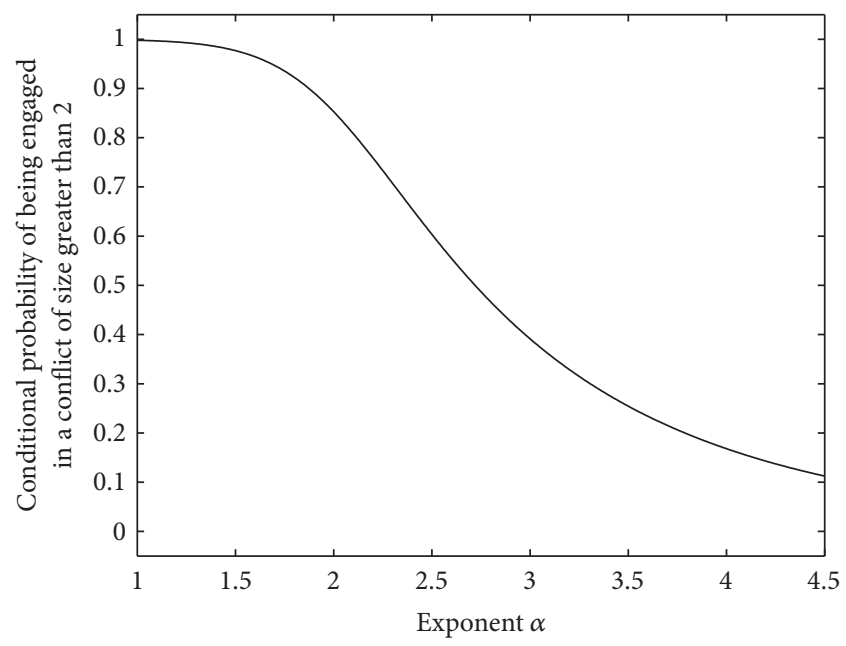

FIGURE 2: Conditional probability of the conflict size being greater than 2 , given that an individual is engaged in a conflict.

thinner, making it harder for cooperators to survive through small conflicts.

In the first part of our work, we show that cooperation can be sustained even when individual-level conflicts dominate, as the size of conflicts follows a power law distribution. However, like conventional mathematical models in which group selection might work, this approach further assumes the repeated isolation, mixture, and reisolation of groups. Constant remixing is rather strong and unrealistic requirement. This may be resolved by introducing a proper population structure such as a network topology.

As mentioned above, the evolutionary game theory has used various types of networks to represent social contact structures and reported the eventual survival of cooperation on such networks. In this study, we adopt the scale-free networks as a platform for extensible groups which lead to varying size conflicts and collective engagements. A link between two nodes in this model represents potential alliance against an opponent, rather than pairwise competition. Introducing network structures into conflict formation enables (1) a natural explanation why conflicts occur according to a power law distribution and (2) flexible groupings while individuals keep their fixed contact relations. To the author's knowledge, the mechanism behind a power law of conflicts has never been studied in relation to human networks. In addition, the scale-free networks we adopted in the paper are not networks representing general contacts or conflicts, but rather networks representing potential alliances. As a result, the power law exponent for the conflict distribution is different from that of the scale-free networks.

\section{Conflicts at Various Scales}

2.1. Model. Suppose there is a series of conflicts among $N$ persons who have either cooperative or selfish strategies. 
Each conflict engages two groups of randomly chosen persons. In our first model, we assume that two groups are of the same size $k, 1 \leq k \leq k_{\max }$, and the size of a conflict $x=2 k$ follows a power law distribution (1). The outcome of a conflict depends on the number of cooperators in the groups; if $k>k_{c}$ for some $k_{c} \geq 2$, the group that has more cooperators than the other is bound to win. This can be justified as the unselfish people are willing to cooperate and provide their resources for their group. However, if the conflict is small or just an interpersonal dispute, $k<k_{c}$, it is assumed that the selfish-dominant group/person is subject to win.

After each conflict, the persons may change their strategies according to the result. The rule for strategy adaptation is that those who are responsible for the group's defeat change their strategy with the adaptation rate $r$. In a group-group conflict, the selfish persons in the defeated group change their strategy to cooperation. On the contrary, a cooperator who lost a small conflict of size $k<k_{c}$ may turn selfish with the probability $r$. In addition to this rule, we also assume that the people randomly change their strategy with a small mutation rate, $q$, between conflicts.
Figure 3 shows a sample simulation of the model consisting of $N=1,000$ people. Initial distributions of cooperative/selfish people are even. After 10,000 sequential conflicts whose sizes follow the power law with $\alpha=3$, the total number of the cooperators reduces to around 250. Here, the parameters $k_{c}=2, k_{\max }=500, r=1$, and $q=10^{-10}$ are used.

2.2. Distribution of Cooperators in Equilibrium. Since our model is a Markov chain, we first seek the corresponding transition matrix $W \in R^{(N+1) \times(N+1)}$, where $W_{n, m}$ is the probability of moving from the state of $m$ cooperators to $n$ cooperators in one-time step. One can see that $W$ is decomposed as two transitional matrices $P$ and $Q$ as

$$
W=Q P .
$$

Here $P$ is the transition matrix for the change through a conflict and $Q$ is for the change by mutations. For simplicity, we set the adaptation rate $r=1$. Then the matrix $P$ is evaluated as

$$
P_{n, m}=P(n \mid m)= \begin{cases}\sum_{k=k_{m, n}}^{k_{\max }} p_{\mathrm{cft}}(2 k) \sum_{i=0}^{n-m} p(m-n+k, m-n+i+k \mid m, k) & \text { if } m<n \\ \sum_{k=1}^{k_{\mathrm{ath}}-1} p_{\mathrm{cft}}(2 k) \sum_{i=0}^{m-n-1} p(m-n, i \mid m, k) & \text { if } m>n \\ \sum_{k=1}^{k_{\max }} p_{\mathrm{cft}}(2 k) \sum_{i=0}^{m / 2} p(i, i \mid m, k) & \text { if } m=n,\end{cases}
$$

where $k_{m, n}=\max (2, n-m)$. Here, $p\left(m_{1}, m_{2} \mid m, k\right)$ denotes a probability that when the total number of cooperators is $m$, two randomly chosen $k$-size groups have $m_{1}$ and $m_{2}$ cooperators, respectively. This can be obtained as

$$
\begin{aligned}
& p\left(m_{1}, m_{2} \mid m, k\right) \\
& =\frac{2\left(\begin{array}{c}
m \\
m_{1}
\end{array}\right)\left(\begin{array}{c}
N-m \\
k-m_{1}
\end{array}\right)\left(\begin{array}{c}
m-m_{1} \\
m_{2}
\end{array}\right)\left(\begin{array}{c}
N-m+m_{1}-k \\
k-m_{2}
\end{array}\right)}{\left(\begin{array}{c}
N \\
k
\end{array}\right)\left(\begin{array}{c}
N-k \\
k
\end{array}\right)}, \\
& \text { if } m_{1} \neq m_{2} .
\end{aligned}
$$

In case of $m_{1}=m_{2}$, it is the half of (4).

$$
Q_{n, m}=Q(n \mid m)= \begin{cases}\sum_{i=0}^{\min (m, N-n)}\left(\begin{array}{c}
m \\
i
\end{array}\right)\left(\begin{array}{c}
N-m \\
n-m+i
\end{array}\right) q^{n-m+2 i}(1-q)^{N+m-n-2 i} & \text { if } m<n \\
\sum_{i=0}^{\min (n, N-m)}\left(\begin{array}{c}
N-m \\
i
\end{array}\right)\left(\begin{array}{c}
m \\
m-n+i
\end{array}\right) q^{m-n+2 i}(1-q)^{N-m+n-2 i} & \text { if } m \geq n .\end{cases}
$$

It is clear that $W$ is a positive Markov matrix, as any two states $m$ and $n$ are directly connected with strictly positive transition probability $W_{n, m}>0$. The Perron-Frobenius theorem for a positive Markov matrix states that 


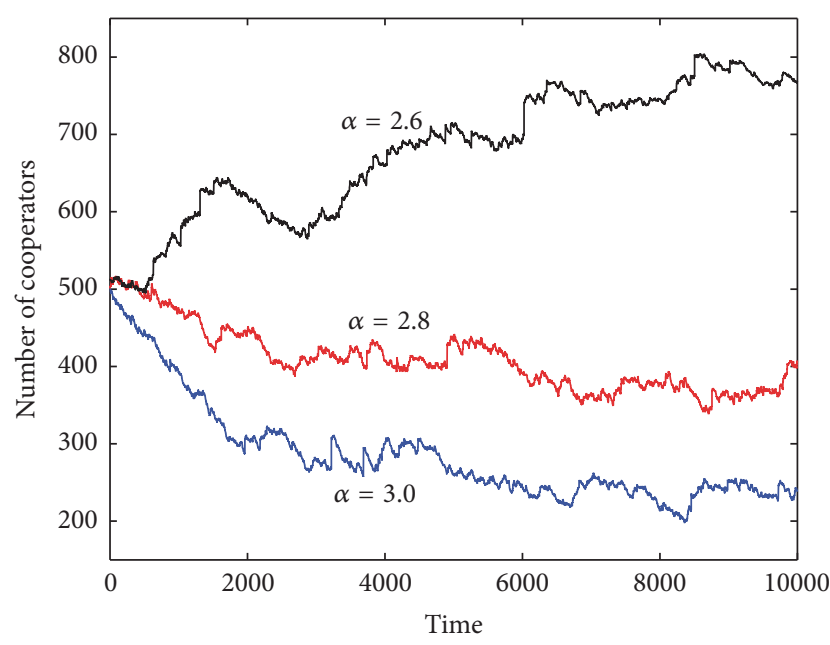

Figure 3: A single sample run of Monte Carlo simulation: the number of cooperators is initially half of the 1,000 population and soon decreases through a series of conflicts which is power law distributed in size with $\alpha=3$.

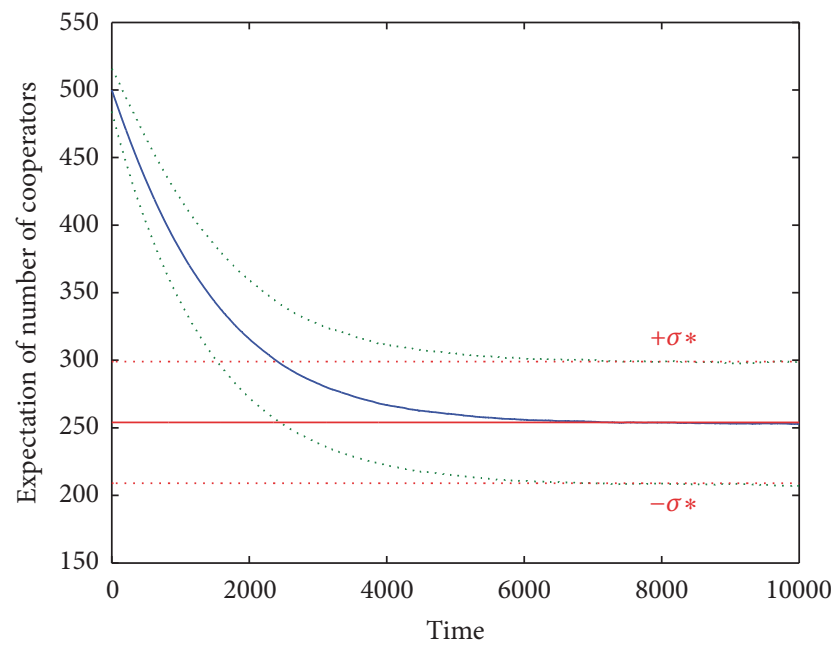

FIGURE 4: Time evolution of the number of the cooperators: the graph (in solid blue) is the ensemble average of 100,000 times of the Monte Carlo realizations with $\alpha=3$. The number of the cooperators converges to the equilibrium (in solid red) which is analytically evaluated in (6).

there exists a unique stationary probability distribution $\mathbf{p}$ satisfying

$$
\mathbf{p}=W \mathbf{p}
$$

and moreover,

$$
\lim _{t \rightarrow \infty} W^{t}=[\mathbf{p}|\mathbf{p}| \cdots \mid \mathbf{p}]
$$

The number of the cooperators in the equilibrium can be obtained from analysis in (6) and (7). The mean is computed from the equilibrium distribution $\mathbf{p}$ and compared to the result from the Monte Carlo simulations in Figure 4. Here the

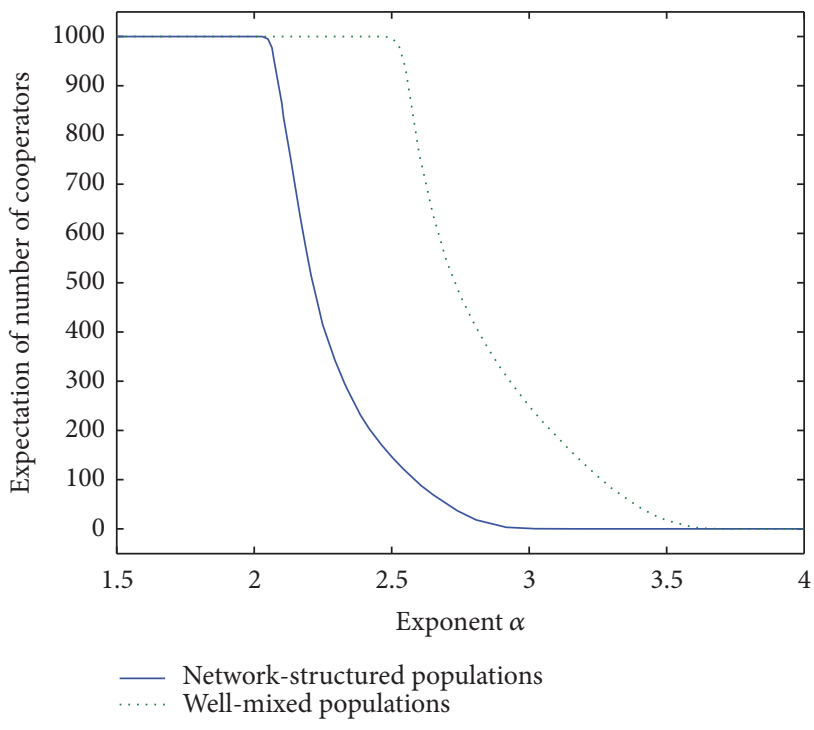

FIGURE 5: The number of the cooperators in the equilibrium according to the power law exponent $\alpha$. The dotted line represents, for a value of $\alpha$ ranging from 1.5 to 4 , how many cooperators out of 1,000 population remain in the end. The results are obtained from an ensemble average of 100,000 Monte Carlo realizations. The solid line illustrates the mean number of the cooperators when the population is structured on a scale-free network. See Section 3.

same parameters, $N=1,000, \alpha=3, k_{c}=2, k_{\max }=50, r=1$, and $q=10^{-10}$, are used as in the setting for Figure 3 .

The dotted graph in Figure 5 shows how the mean of the number of the cooperators in the equilibrium changes according to the exponent $\alpha$. The phase transition occurs at around 2.5 and the mean starts to drop to zero as $\alpha$ approaches 3.5. This may be interpreted as a very favourable signal for the cooperators, since most estimates for $\alpha$ based on the empirical data of human conflicts range from 1.5 to 2.5 . However, referring to the graph in Figure 2, one can see that if more than $70 \%$ of the disputes that each person undergoes are interpersonal ones, the absolute number of people turns to selfish.

\section{Conflicts in Networked Population}

3.1. Model. The earlier model is based on the homogeneous population without any fixed group structures. The assumption based on constant mixing of the population after each conflict is not quite realistic, especially for largescale disputes. In addition, the survival of cooperation in the model essentially depends on the conditional probability of an individual's being engaged in a group-group conflict, which is described in Figure 2. Thus cooperation in this setting does not necessarily require power law distributed conflicts, as long as large conflicts do not occur too scarcely.

In the second model, we introduce a network structure to indicate people's relations, keeping the same win-loss and adaptation rule from the previous model. Each node of the network represents an individual and the link connecting 


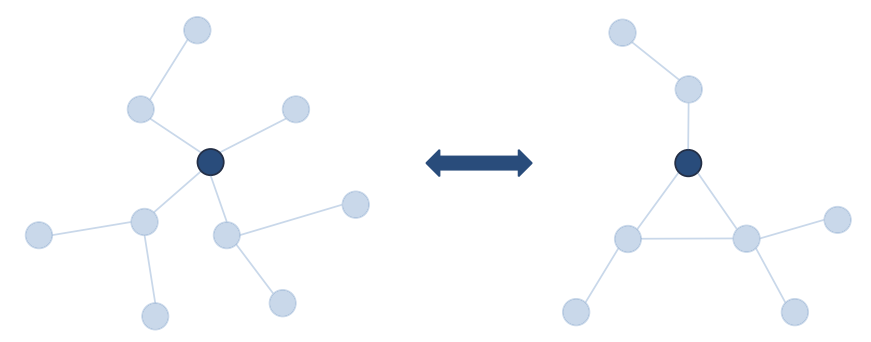

(a) Conflict between individuals

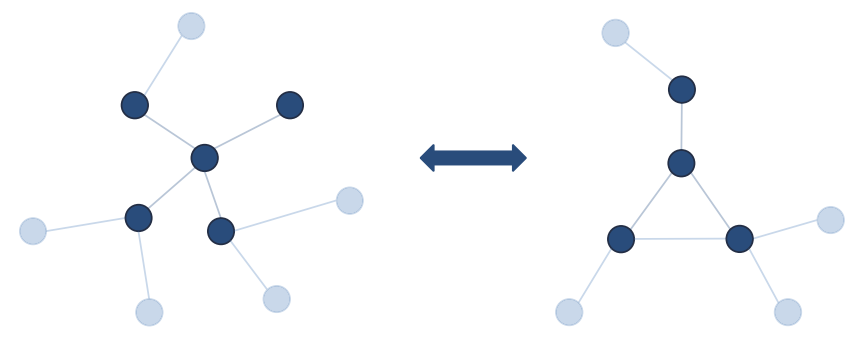

(b) Conflict extended over a network

FIGURE 6: Formation of conflicts of various sizes over a network. A dispute initiated between two persons (illustrated in (a)) may develop into a larger conflict (in (b)), following the networks. This escalation occurs with the probability $\beta$ and may repeat several times, creating conflicts between two arbitrary clusters of various sizes.

two nodes represents their companionship which possibly develops into alliance in a conflict. We assume that a group is essentially an alliance to win a dispute and competitive alliance formation is responsible for the group size distribution. Then it is important to see on what kind of structure alliances grow. Here, as such structure, we suggest networks that represent potential alliances between individuals. On the networks, alliances are made between neighbors while conflicts occur between relative strangers. Accordingly, we set that every conflict initiates as a hostile event between persons at two arbitrary nodes (which are generally not neighboring each other).

The dispute could end up just as a interpersonal conflict as in Figure 6(a), or, it may develop into a larger fight and gets more people involved who are next to them as in Figure 6(b). If such spreading occurs $l$ times, the eventual size of the conflict will be sum of the two $l$ th neighborhoods of the original nodes. We set this conflict escalation rate as the probability $\beta$. Note that two groups are not necessarily the same size, which is more realistic when modeling large group disputes. The assumption here is that two groups experience the same moments of enlarging over the networks and therefore the group sizes are expected to agree only in the mean. Also note that even the same person can belong to a different group in a different conflict, depending on who is engaged in the fight among his/her neighbors.

Modeling conflicts over a networked structured population enables flexible groupings while individuals keep their fixed contact relations. It has been empirically observed that scale-free networks frequently occur in many complex real-world systems, especially in social networks [39]. In our model, the scale-free structure is adopted to represent collective engagements and flexible (but not random) grouping among people. It provides a natural explanation why the size of conflicts exhibits a power law distribution. From the fact that the degree distribution of the scale-free network is a power law, one can derive that conflicts extended over such network in the above manner are bound to a similar distribution. Indeed, they follow a power law with the exponent

$$
\alpha \approx-\frac{\ln \beta}{\ln \gamma}
$$

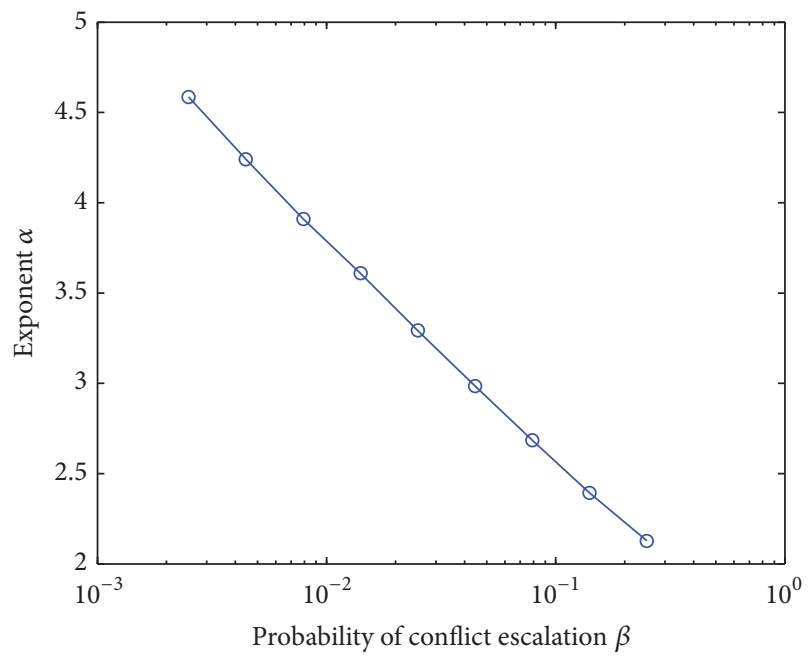

FIGURE 7: Relation between the conflict escalation rate and the power law exponent of the conflict size for a scale-free network. Once the topology of the network is fixed, the conflict escalation rate $\beta$ determines the distribution of the conflict size as in (8).

where $\gamma$ is the mean number of the second next neighbors of the nodes in the network. This relation is shown in Figure 7.

3.2. Survival of Cooperation over Scale-Free Networks. Once we assign a fixed social structure into a population model, the situation usually turns against the cooperators, compared to the homogeneously mixed population.

In the second model, we use scale-free networks with 1,000 nodes each representing an individual. The links are generated by the preferential attachment scheme, that is, the standard procedure to create scale-free networks [40]. Since the scale-free networks are characterized by the existence of hubs, we are especially interested in how they behave in the system. Are the hubs more cooperative or more selfish?

Figure 5 depicts the ensemble average of the number of the cooperative people in the solid line. The same parameters are used and the exponent $\alpha$ is converted from the escalation rate $\beta$ according to Figure 7 . The graph is shifted to the left from that of the well-mixed population, making it hard for the cooperation to flourish. If interpersonal conflicts 


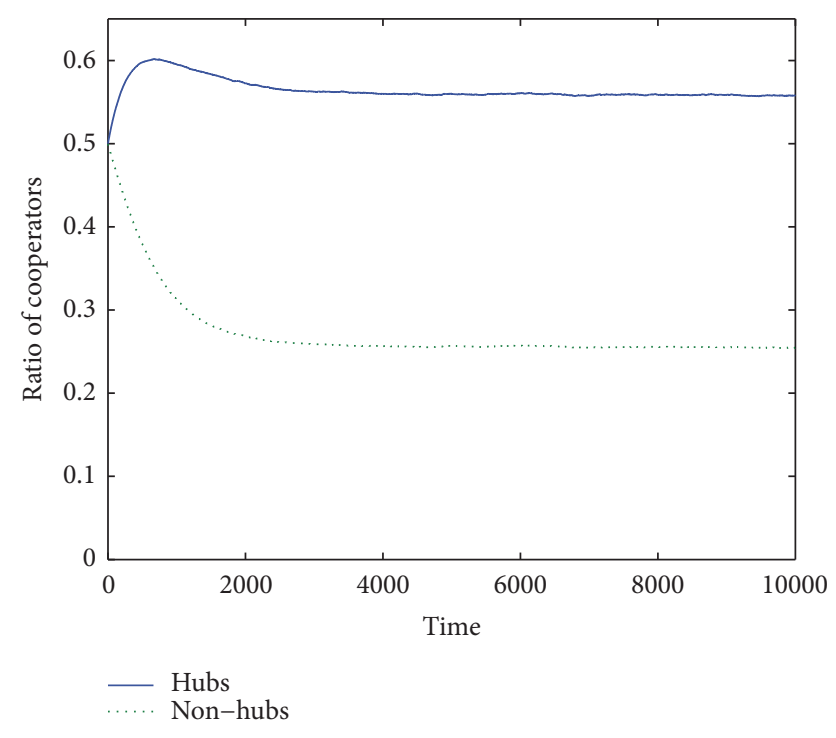

Figure 8: Time evolution of the mean ratio of the cooperators according to the types of nodes. More than half of the people at the hubs remain cooperative, while over two-thirds of the people at nonhubs quickly turn selfish. The results are obtained from 100,000 times of Monte Carlo simulations with $\alpha=3$.

become more than $50 \%$ of the conflicts that each individual undergoes, then most of them choose to be selfish. However, the graph holds the same qualitative behaviour and the cooperation manages to be sustained for the exponent $\alpha$ from 2 to 2.5, which is still in the range reported from the many empirical researches [35-38]. Considering the population has a fixed network structure, one can see that the preservation of cooperation does not necessarily require either constant reorganization of the groups or occasional member exchanges between them, unlike the conventional group selection theory.

It is notable that individuals at the hubs of the networks play a key role in sustaining cooperation. Note that, since the hubs have more links to other nodes, the persons at the hubs are more likely to be engaged in large-scale conflicts rather than 1-1 fights. Therefore they tend to keep themselves cooperative even when most people elsewhere fall to selfishness. Figure 8 compares the cooperators at the hubs (the nodes with 10 or more links) to the cooperators at other nodes. While the mean ratio of the cooperators at normal nodes converges to around 25\%, more than half of the hubs remain unselfish. They resist a general tendency and become sources of cooperation. In this regard, our common sense about the relation between altruistic cooperation and popularity, "good people make hubs," is indeed true and the other way around; hubs make people good.

\section{Conclusion}

Since the cooperators use their resources to help their group win, they are put at a disadvantage in a man-to-man fight. However, even when such interpersonal disputes dominate, the cooperators can survive or even flourish, as the size distribution of conflicts follow a power law distribution. One can derive the convergent equilibrium distribution of the cooperative people in the well-mixed population.

The conventional cooperation model based on the naive group selection has been criticised in that it requires frequent mixing between the groups and excludes long lasting social structures. In this work, we assume that people's companionship is structured as scale-free networks and their conflicts can develop into a larger conflict, propagating over the networks. This leads to a natural explanation of why human conflicts exhibit a power law distribution and how cooperation successfully continues to exist. The population with a fixed structure is less favourable to the cooperators than the homogeneously mixed population. This is seemingly contradictory to classical game-theoretical models where the scale-free structure promotes cooperation $[18,19,25]$. However, our model is different in that the nodes in the neighbor are not competitors to play with but rather potential allies to cope with external disputes. One can see that cooperation still survives as long as the corresponding power law exponent is in the suitable range.

Since the scale-free networks are characterized by the existence of hubs, we are interested in their behaviour in the system. It is important that the individuals at the hubs get involved in large conflicts more frequently and therefore tend to remain unselfish. The observation that the hubs become sources of cooperation may give meaningful implication on the role of the hubs in the social networks.

\section{Disclosure}

The funders had no role in study design, data collection and analysis, decision to publish, or preparation of the manuscript.

\section{Competing Interests}

The author declares that there is no conflict of interests regarding the publication of this paper.

\section{Acknowledgments}

This work was supported by the National Research Foundation of Korea grant funded by the Korean Government (NRF2015S1A5A2A03049830).

\section{References}

[1] R. L. Trivers, "The Evolution of Reciprocal Altruism," The Quarterly Review of Biology, vol. 46, no. 1, pp. 35-57, 1971.

[2] M. A. Nowak, "Five rules for the evolution of cooperation," Science, vol. 314, no. 5805, pp. 1560-1563, 2006.

[3] D. S. Wilson, "A theory of group selection," Proceedings of the National Academy of Sciences of the United States of America, vol. 72, no. 1, pp. 143-146, 1975.

[4] R. Boyd and P. J. Richerson, "Group selection among alternative evolutionarily stable strategies," Journal of Theoretical Biology, vol. 145, no. 3, pp. 331-342, 1990. 
[5] A. Traulsen and M. A. Nowak, "Evolution of cooperation by multilevel selection," Proceedings of the National Academy of Sciences of the United States of America, vol. 103, no. 29, pp. 10952-10955, 2006.

[6] W. Hamilton, "The genetical evolution of social behaviour. I," Journal of Theoretical Biology, vol. 7, no. 1, pp. 1-16, 1964.

[7] J. Seger, "Cooperation and conflict in social insects," in Behavioural Ecology, J. R. Krebs and N. B. Davies, Eds., pp. 338373, Blackwell, Oxford, UK, 3rd edition, 1991.

[8] J. Tanimoto, "A study of indirect reciprocity involving a reputation system or a simple tag system in a one-shot, multi-player game," BioSystems, vol. 90, no. 3, pp. 856-869, 2007.

[9] A. Traulsen, D. Semmann, R. D. Sommerfeld, H.-J. Krambeck, and M. Milinskib, "Human strategy updating in evolutionary games," Proceedings of the National Academy of Sciences of the United States of America, vol. 107, no. 7, pp. 2962-2966, 2010.

[10] E. Fehr and K. M. Schmidt, "A theory of fairness, competition, and cooperation," The Quarterly Journal of Economics, vol. 114, no. 3, pp. 817-868, 1999.

[11] E. Fehr and S. Gächter, "Altruistic punishment in humans," Nature, vol. 415, pp. 137-140, 2002.

[12] V. Capraro, "A model of human cooperation in social dilemmas," PLoS ONE, vol. 8, no. 8, Article ID e72427, 2013.

[13] G. E. Bolton and A. Ockenfels, "ERC: a theory of equity, reciprocity, and competition," The American Economic Review, vol. 90, no. 1, pp. 166-193, 2000.

[14] G. Charness and M. Rabin, "Understanding social preferences with simple tests," Quarterly Journal of Economics, vol. 117, no. 3, pp. 817-869, 2002.

[15] D. G. Rand, A. Peysakhovich, G. T. Kraft-Todd et al., "Social heuristics shape intuitive cooperation," Nature Communications, vol. 5, article 3677, 2014.

[16] M. Tomasello, A. P. Melis, C. Tennie, E. Wyman, and E. Herrmann, "Two key steps in the evolution of human cooperation. The interdependence hypothesis," Current Anthropology, vol. 53, no. 6, pp. 673-692, 2012.

[17] A. Guazzini, D. Vilone, C. Donati, A. Nardi, and Z. Levnajić, "Modeling crowdsourcing as collective problem solving," Scientific Reports, vol. 5, article 16557, 2015.

[18] M. A. Nowak and R. M. May, "Evolutionary games and spatial chaos," Nature, vol. 359, no. 6398, pp. 826-829, 1992.

[19] C. P. Roca, J. A. Cuesta, and A. Sánchez, "Evolutionary game theory: temporal and spatial effects beyond replicator dynamics," Physics of Life Reviews, vol. 6, no. 4, pp. 208-249, 2009.

[20] M. Perc and A. Szolnoki, "Social diversity and promotion of cooperation in the spatial prisoner's dilemma game," Physical Review E, vol. 77, no. 1, Article ID 011904, 2008.

[21] M. Perc, "Coherence resonance in a spatial prisoner's dilemma game," New Journal of Physics, vol. 8, article 22, 2006.

[22] M. Perc, "Transition from Gaussian to Levy distributions of stochastic payoff variations in the spatial prisoner's dilemma game," Physical Review E-Statistical, Nonlinear, and Soft Matter Physics, vol. 75, no. 2, Article ID 022101, 2007.

[23] F. C. Santos and J. M. Pacheco, "Scale-free networks provide a unifying framework for the emergence of cooperation," Physical Review Letters, vol. 95, no. 9, Article ID 098104, 2005.

[24] S. Assenza, J. Gómez-Gardeñes, and V. Latora, "Enhancement of cooperation in highly clustered scale-free networks," Physical Review E, vol. 78, no. 1, Article ID 017101, 2008.
[25] J. Gómez-Gardeñes, M. Campillo, L. M. Floría, and Y. Moreno, "Dynamical organization of cooperation in complex topologies," Physical Review Letters, vol. 98, no. 10, Article ID 108103, 2007.

[26] J. Gomez-Gardenes, M. Romance, R. Criado, D. Vilone, and A. Sanchez, "Evolutionary games defined at the network mesoscale: the Public Goods game," Chaos, vol. 21, no. 1, 2011.

[27] J. Peña and Y. Rochat, "Bipartite graphs as models of population structures in evolutionary multiplayer games," PLOS ONE, vol. 7, no. 9, Article ID e44514, 2012.

[28] M. Perc and A. Szolnoki, "Coevolutionary games-a mini review," BioSystems, vol. 99, no. 2, pp. 109-125, 2010.

[29] M. Perc, J. Gómez-Gardeñes, A. Szolnoki, L. M. Floría, and Y. Moreno, "Evolutionary dynamics of group interactions on structured populations: a review," Journal of the Royal Society, Interface/The Royal Society, vol. 10, no. 80, 2013.

[30] L.-E. Cederman, "Modeling the size of wars: from billiard balls to sandpiles," American Political Science Review, vol. 97, no. 1, pp. 135-150, 2003.

[31] C. Cioffi-Revilla and M. Midlarsky, "Highest magnitude warfare: power laws, scaling, and fractals in the most letha international and civil wars," in The Scourge of War, P. Diehl, Ed., The University of Michigan Press, Ann Arbor, Miss, USA, 2003.

[32] J. S. Levy and T. C. Morgan, "The frequency and seriousness of war: an inverse relationship?" Journal of Conflict Resolution, vol. 28, no. 4, pp. 731-749, 1984.

[33] L. F. Richardson, "Variation of the frequency of fatal quarrels with magnitude," Journal of the American Statistical Association, vol. 43, no. 244, pp. 523-546, 1948.

[34] R. Overill and J. Silomon, "Single and double power laws for cyber-crimes," Journal of Information Warfare, vol. 10, no. 3, pp. 29-36, 2011.

[35] J. A. Friedman, "Using power laws to estimate conflict size," Journal of Conflict Resolution, vol. 59, no. 7, pp. 1216-1241, 2015.

[36] A. Clauset and M. Young, "Scale invariance in global terrorism," https://arxiv.org/abs/physics/0502014.

[37] N. Johnson, M. Spagat, J. Restrepo et al., From Old Wars to New Wars and Global Terrorism, http://xxx.lanl.gov/abs/physics/ 0506213.

[38] N. Johnson, M. Spagat, J. Restrepo et al., "Universal patterns underlying ongoing wars and terrorism," http://xxx.lanl.gov/ abs/physics/0605035.

[39] G. Caldarelli, Scale-Free Networks, Oxford University Press, Oxford, UK, 2007.

[40] A.-L. Barabasi and R. Albert, "Emergence of scaling in random networks," American Association for the Advancement of Science. Science, vol. 286, no. 5439, pp. 509-512, 1999. 


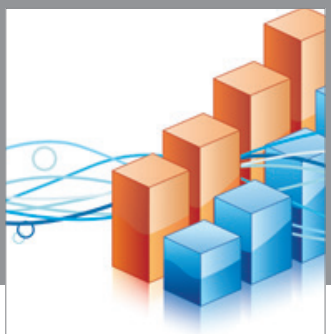

Advances in

Operations Research

vatem alat4

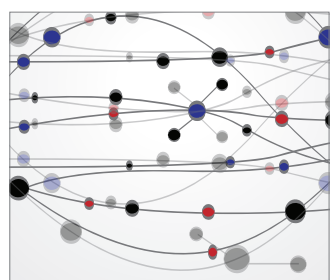

\section{The Scientific} World Journal
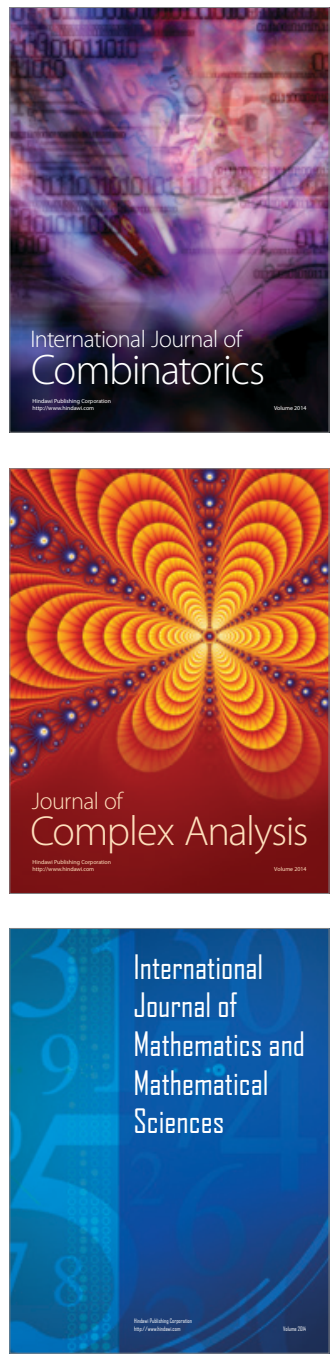
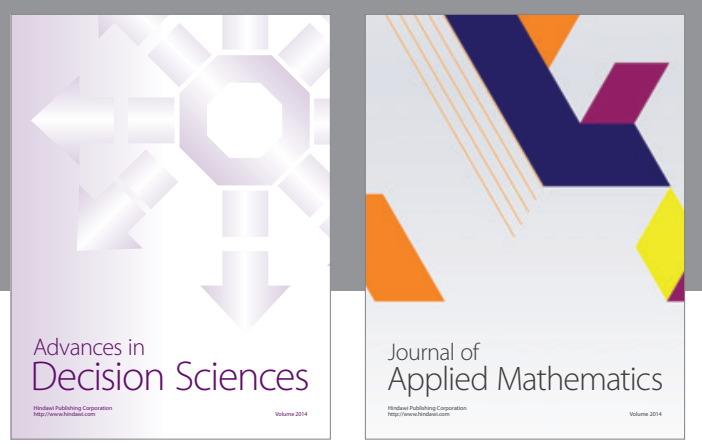

Algebra

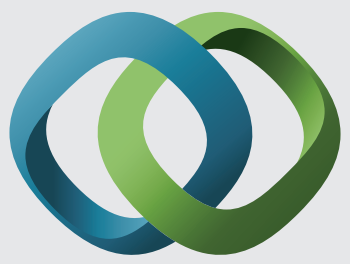

\section{Hindawi}

Submit your manuscripts at

https://www.hindawi.com
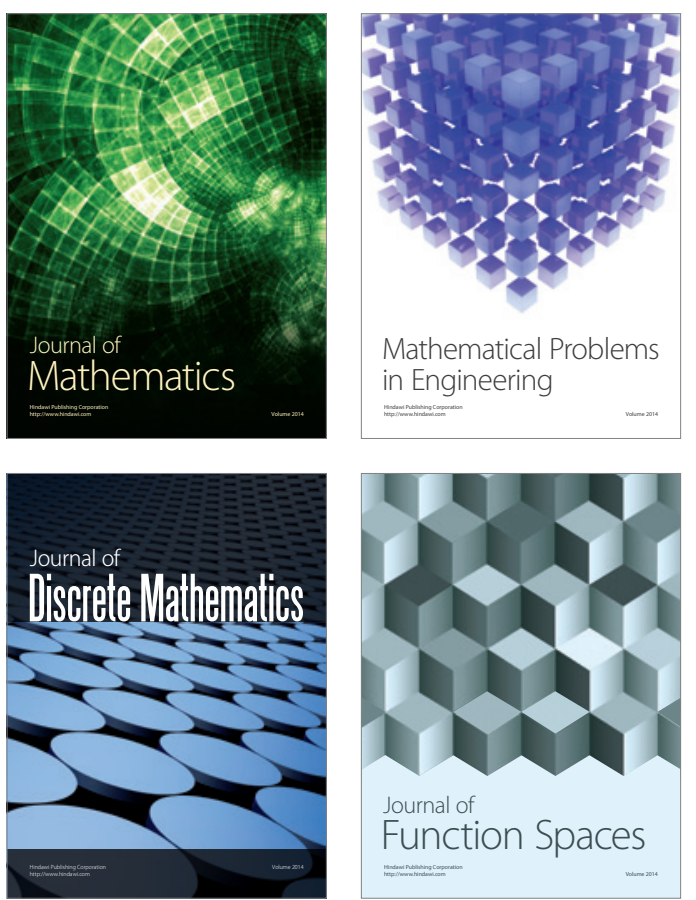

Mathematical Problems in Engineering
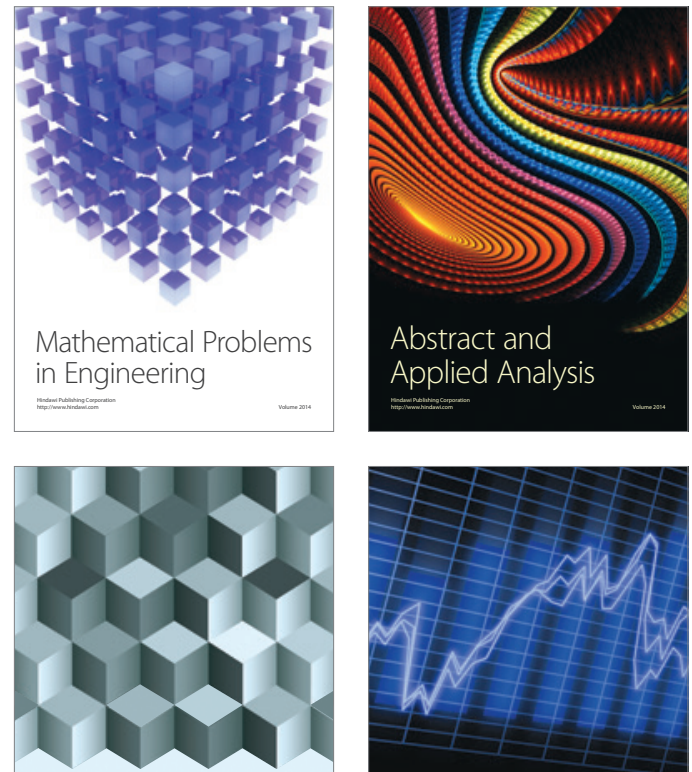

Journal of

Function Spaces

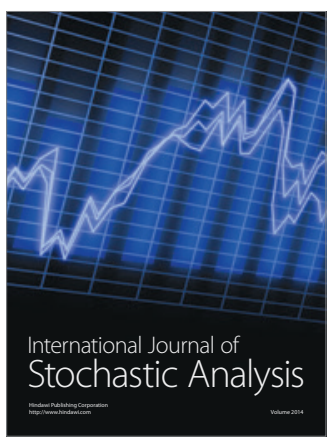

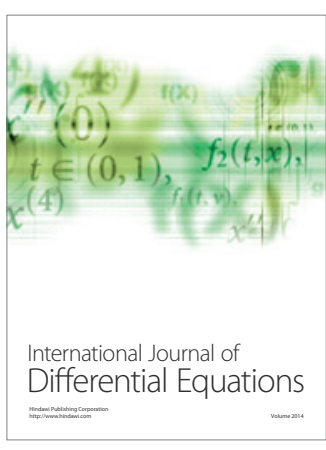
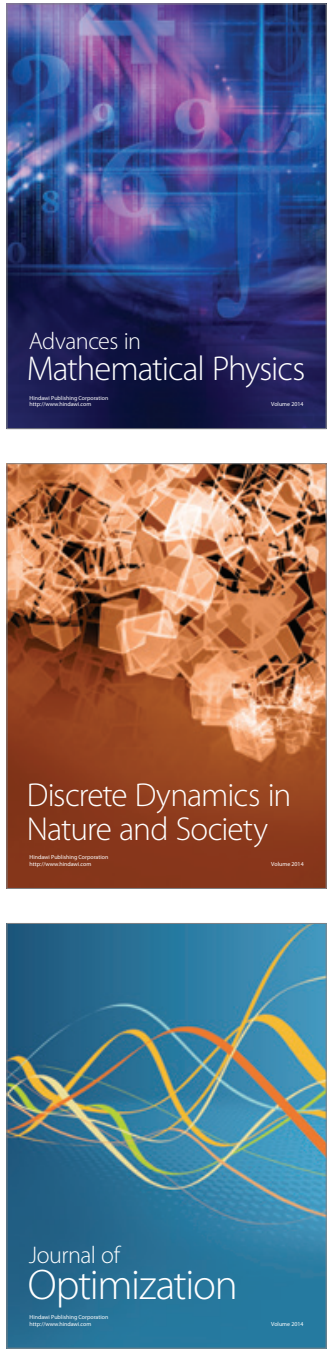